\title{
CoVId19 EN CHILE. A CERCAMIENTO A LAS PERCEPCIONES $Y$ EXPERIENCIAS DE LA PANDEMIA EN MEDIO DE UNA CRISIS SOCIOPOLÍTICA ${ }^{1}$
}

\section{Daniela Henríquez Encamilla ${ }^{2}$}

daniela.henriquez@ce.ucn.cl

Universidad Católica del Norte, Chile.

\section{Yanina Inostroza Jiménez $z^{3}$}

yanina.inostroza@ucn.cl

Universidad Católica del Norte, Chile

\begin{abstract}
El artículo reflexiona sobre las percepciones y experiencias de la pandemia por COVID19 en Chile en el contexto de la crisis sociopolítica que vive el país. El análisis se hace a partir de los resultados de dos levantamientos de información realizados en la Región de Coquimbo y constituye un acercamiento exploratorio de la pandemia, en el contexto de estallido social, a partir de los conceptos de riesgo y confianza. Se concluye que la desconfianza se agudiza, producto de una percepción de desamparo frente a las amenazas de la pandemia, especialmente respecto a las instituciones políticas democráticas.
\end{abstract}

Palabras Claves: Pandemia - estallido social - riesgo - confianza

1 Agradecemos la colaboración del equipo del Instituto de Políticas Públicas y de la Escuela de Ciencias Empresariales UCN Coquimbo y especialmente a las personas participantes en los levantamientos de información realizados.

2 Socióloga, estudiante de Magíster en Desarrollo Económico. Instituto de Políticas Públicas, Universidad Católica del Norte

3 Socióloga. Instituto de Políticas Públicas, Universidad Católica del Norte. 


\section{CoVId19 in Chile. ApProACH tO THE PERCEPTIONS} AND EXPERIENCES OF THE PANDEMIC WITHIN THE CONTEXT OF A SOCIO-POLITICAL CRISIS

The article reflects on the perceptions and experiences about COVID19 pandemic in Chile in the context of the socio-political crisis in the country. The analysis is carried out from the results of two surveys carried out in the Coquimbo Region and constitutes an exploratory approximation about the pandemic, in the context of a social outbreak, based on the concepts of risk and trust. We concluded that mistrust become more acute, as a result of a perception of helplessness in the face of the threats of the pandemic, especially regarding democratic political institutions.

Keywords: Pandemic - social outbreak - risk - trust 


\section{Introducción}

La pandemia del COVID 19 debe ser uno de los eventos más relevantes en la historia mundial reciente. A marzo de 2021, el virus ha provocado más de 2,5 millones de muertes en el mundo y más de 116 mil millones de casos confirmados de contagio (World Health Organization, 2021). Además, de acuerdo con el informe anual de las Naciones Unidas (2021), la pandemia ha afectado cada aspecto de la vida moderna y ha sido mucho más que una crisis sanitaria, también ha sido una crisis económica, humanitaria, de derechos humanos y de seguridad.

La región latinoamericana ha sido de las más golpeadas, la pandemia ha agudizado desafios sociales estructurales entre los cuales destacan la desigualdad social, la informalidad laboral, la desprotección social y la pobreza (Comisión Económica para América Latina y el Caribe, 2021). De esta forma, Chile recibe la pandemia en medio de uno de los movimientos sociales más relevantes de su historia reciente. De acuerdo con datos oficiales, más de 4 millones de personas se movilizaron en las calles a partir del 18 de octubre de 2019, fecha del llamado "Estallido Social". Por otro lado, el Instituto de Derechos Humanos ha observado 308 movilizaciones sociales en todo el país hasta febrero de 2020 reportando a su vez 3.764 personas lesionadas y 1.835 vulneraciones de derechos ocurridas en recintos policiales contra personas detenidas en manifestaciones sociales (INDH, 2020). Producto de las diferentes movilizaciones populares, el 15 de noviembre se firma el llamado "Acuerdo por la Paz y la Nueva Constitución" adscrito por diferentes partidos políticos, el cual comprometió "una salida institucional cuyo objetivo es buscar la paz y la justicia social a través de un procedimiento inobjetablemente democrático" (Biblioteca del Congreso Nacional de Chile, 2019:1). Dicho acuerdo marcó el inicio de un proceso constituyente que fue aprobado por el $78 \%$ de los votantes el pasado octubre de 2020 .

Actualmente el país vive un proceso social y político particular, el cual coincide en tiempo y espacio con la pandemia. Ambas situaciones tienen innumerables impactos y complejas relaciones. El presente artículo es un aporte exploratorio para describir las percepciones sobre el impacto de la pandemia de COVID 19 en el contexto chileno, donde se vive una de las crisis sociales y políticas más relevantes de su democracia. Para este objetivo se han utilizado dos fuentes de información primaria, levantamientos realizados durante el año 2020 en la Región de Coquimbo. Por una parte, se utilizan los resultados de una encuesta web no probabilística donde se consultó a las personas respecto al impacto de la pandemia en sus vidas, proceso realizado por el Instituto de Políticas Públicas de la Universidad Católica del Norte, sede Coquimbo (IPP UCN). Por otro lado, se utilizan los resultados de un taller participativo efectuado por la misma institución, el cual, a través de video llamada, tuvo como objetivo conocer las percepciones y experiencias de las personas en este contexto de contingencia sanitaria. El trabajo que se presenta a continuación ha adoptado una metodología mixta, con énfasis en un enfoque cualitativo 
hermenéutico que tiene como propósito principal comprender la realidad social (Ruiz, 2012).

El aporte del presente artículo es poder reflexionar respecto a cómo una sociedad enfrenta el riesgo cuando la confianza, principal herramienta para reducir las complejidades y contingencias, se encuentra en crisis. A partir de los resultados es posible observar que la experiencia de la pandemia en Chile se vive desde la percepción de abandono por parte del Estado y el fortalecimiento del espacio privado y círculo más cercano como únicos sitios de seguridad frente a un sistema exterior de riesgo. Se concluye que el riesgo se consolida como constitutivo de lo social y la desconfianza se profundiza.

\section{Una sociedad del riesgo global}

De acuerdo con Taylor-Gooby y Zinn (2006), las teorías del riesgo pueden clasificarse, principalmente, dependiendo de su ontología y particularidad. De esta forma, las teorías se van a diferenciar dependiendo del grado de independencia entregado a los riesgos y de la importancia otorgada al individuo y/o al colectivo social para su análisis (Taylor-Gooby \& Zinn, 2006). Si bien las oportunidades de articulación entre las diferentes teorías y enfoques no se han agotado, se observa una tendencia a que los enfoques centrados en el individuo cada vez incorporen más aspectos sociales (Taylor-Gooby \& Zinn, 2006).

Douglas junto con Wildavsky (1982) argumentan que las preocupaciones de las personas sobre los riesgos (específicamente desastres ambientales), dependen del tipo de organización social en la cual se desenvuelven y distinguirían tres tipos de organizaciones sociales: la individualista y emprendedora; la jerárquica y burocrática; y la sectaria. Para efectos de este artículo, cabe destacar el primer caso, también denominadas sociedades de mercado, donde los beneficios son adquiridos tomando riesgos individuales y pensando principalmente en el corto plazo. Los individuos perciben que dependen sólo de sí mismos, pagando individualmente el costo de los riesgos que corren (Spickard, 1989). En sintesis, de acuerdo con Douglas y Wildavsky (1982), las percepciones de las personas respecto al riesgo están mediadas por el contexto de sus vidas sociales (Spickard, 1989).

Pero el riesgo no sólo es percibido socioculturalmente, para Beck (1998) el riesgo es, en efecto, constitutivo de la sociedad moderna. Algo relevante de la perspectiva de Beck, es que el riesgo no es un daño producido, no es destrucción en sí misma, sino que es una amenaza de destrucción (Mitjavila, 1999). El riesgo empieza donde la confianza en la seguridad termina, determinando (a través de este espacio entre seguridad y daño o destrucción) el pensamiento y las acciones de los sujetos y generando futuros inciertos en los diferentes espacios vitales (Alemán, 2013). Por lo tanto, la relación con el tiempo y el espacio también se transforma. El futuro y sus posibilidades comienzan a incidir en la toma de decisiones de hoy (Beck, 1998) 
y la mirada ya no puede ser limitada a las fronteras territoriales del Estado Nación, debido a la globalización de los riesgos (Alemán, 2013).

En una sociedad del riesgo, la fuente de nuevos riesgos estaría justamente en el creciente conocimiento (Beck, 1998). La desmonopolización de la ciencia ha permitido reflexionar sobre sus límites y certezas en la medida que la ciencia solo sería capaz de producir información "más o menos" certera (Beck, 1998). Lo anterior tendría también importantes impactos en la política en la medida que ésta asumirá el papel de legitimar democráticamente decisiones que desconoce. En este proceso de toma de decisiones sobre supuestos desconocidos, finalmente, las responsabilidades se diluyen, las responsabilidades son de nadie (Beck, 1998). En este sentido, la sociedad del riesgo es una sociedad de constantes crisis donde "los peligros los produce la industria, los exterioriza la economía, el sistema legal los individualiza y la política los hace parecer inofensivos" (Beck, 1998:509), frente a lo cual se socava el poder y la credibilidad de las instituciones.

\section{La confianza en una sociedad en riesgo}

Dentro de la literatura se pueden encontrar, principalmente, dos enfoques (Saldaña y Pineda, 2019). Por una parte, un enfoque racional institucional y por otra parte un enfoque culturalista. Respecto al enfoque racional institucional, la confianza sería el grado de satisfacción de las personas respecto a las instituciones en un momento determinado y en la medida que éstas entregan ciertas garantías relacionadas a derechos, libertades y seguridades políticas y económicas (Saldaña y Pineda, 2019). En este sentido, para Morales (2008) existe una estrecha relación entre la confianza institucional, la satisfacción con la democracia y su legitimidad. En este enfoque racional institucional, el desempeño económico nacional y la percepción sobre la corrupción serían variables relevantes que impactan en la confianza hacia las instituciones (Saldaña y Pineda, 2019). Por otra parte, para el enfoque culturalista, la confianza política se explica por la aprehensión a normas y valores que se incorporan a través de procesos de socialización, lo cual se conoce como confianza social (Saldaña y Pineda, 2019). En este sentido, la confianza interpersonal se relaciona con la confianza cívica en la medida que, conociendo a otros, las personas serían más proclives a tener confianza social (Urteaga, 2013). De acuerdo con Putnam (1994) la confianza social se genera a través de la experiencia con otros, en grupos, especialmente en la participación de organizaciones. Por otro lado, se puede destacar el importante aporte de Easton (1975), para quien la confianza es parte del apoyo político, específicamente del apoyo difuso que se entiende como las actitudes hacia una comunidad política, régimen o autoridad según lo que representa y no respecto a lo que hace (Tamayo \& Carrillo, 2003). La presencia de confianza significará que las personas sientan atendidos sus intereses incluso sin que las autoridades sean supervisadas, una satisfacción simbólica con el proceso que resulta de la experiencia en el tiempo (Easton, 1975). 
La confianza siempre involucrará un riesgo, una posibilidad de duda (Lewis \& Weigert, 1985). Sin embargo, al mismo tiempo, es a través de la confianza que es posible reducir las complejidades en la imposibilidad de desarrollar planes de acción que tomen en cuenta todos los posibles escenarios futuros contingentes. La confianza es, finalmente, lo que permite que frente al futuro contingente no se paralice la acción (Lewis \& Weigert, 1985). En otras palabras, la confianza es la que permite reducir las complejidades en una sociedad donde la vivencia del riesgo presupone una seguridad perdida, una confianza rota (Beck, 1998).

\section{3. "Estallido social"}

La crisis sanitaria se despliega en un contexto de importantes movilizaciones sociales en todo el país que comienzan con el llamado "Estallido Social" y que podría denominarse como un "momento constituyente" (Choudhry, 2008; PNUD, 2015). El análisis de las causas y características de estas movilizaciones sociales (que continúan) tienen larga data en la historia y en la literatura y probablemente seguirán siendo objeto de estudio en el futuro. Un análisis completo sobrepasaría los alcances de este artículo, sin embargo, es posible destacar algunos aspectos.

La democracia en Chile viviría una contradicción en la medida que sus indicadores económicos son los mejores de la región, pero al mismo tiempo la participación electoral y la representación tienen importantes debilidades (Morales, 2020). Así mismo lo señala Miranda (2018) quien para entonces, observaba que pese a la calidad de la democracia chilena reportada por diferentes indicadores, el país mostraba "una preocupante combinación de una sociedad altamente politizada con un sistema democrático, y en particular un sistema de partidos políticos deslegitimizado" (Miranda, 2018:98). En este sentido Miranda destaca que las demandas sociales pasaron del espacio electoral a las manifestaciones públicas, donde no solamente se demandan libertades políticas y civiles, sino que también derechos económicos y sociales. Al respecto Jiménez-Yáñez (2020) destaca las sucesivas manifestaciones previas al 18 de octubre donde la ciudadanía habría perdido la confianza en la clase política y donde los gobiernos habrían sido incapaces de escuchar y empatizar con las demandas (Jiménez-Yáñez, 2020). Brieba (2020), por otro lado, señala que las tres principales demandas sustantivas del estallido social serían: Dignidad, relacionada a necesidades económicas objetivas y mejores servicios ofrecidos por el Estado. Una demanda en contra del abuso, como una sensación de impunidad de quienes tienen poder y una demanda de mayor igualdad multidimensional (Brieba, 2020).

Por su parte Morales (2020), en términos más estructurales, identifica cuatro crisis que podrían explicar la revuelta social. Una crisis de participación, una crisis de representación, una crisis de confianza y una crisis de probidad. Respecto a la primera se puede destacar la pérdida de ésta en las instituciones democráticas que también afecta a las policías e iglesia que "históricamente funcionaron como pegamento social” (Morales, 2020:18). La crisis conviviría además con una baja confianza 
interpersonal y un aumento en la percepción de conflicto social (Morales, 2020), destacando además la crisis de probidad que habría acelerado y agudizado la caída de la confianza a través de la acumulación de escándalos políticos y económicos que afectaron las condiciones de vida de las personas y acentuaron el malestar.

La caída en la confianza en los partidos políticos no es algo que afecte solamente a Chile. Según Albala y Vieira (2014) la llamada "crisis de los partidos políticos" es algo que se puede observar desde los años noventa en América Latina. Por otro lado, a los factores mencionados como plausibles causas del estallido social, se debe agregar lo que Heiss denomina como el "problema constitucional", en la medida que los principales elementos de la crisis de legitimidad se derivan de los límites a la democracia que impuso la Constitución de 1980, pese a sus reformas (Heiss, 2017).

Son múltiples factores que, como señala Araujo (en Araujo y Benítez, 2019), van más allá de demandas insatisfechas y se relacionarían con procesos de individuación. Según Araujo (en Araujo y Benítez, 2019) el estallido social se relaciona con anclajes existenciales íntimos, construidos colectivamente, es una "recomposición de las normas que regulan las relaciones sociales y la vida en común" (Araujo \& Benítez, 2019:154), tanto entre las personas como también con las instituciones. Canales (en Hopenhayn, 2020) también va a coincidir en que el estallido social es mucho más que una demanda de derechos o bienes, sino que involucra una subjetividad construida que no se corresponde con el orden social cotidiano donde se despliega. Para Canales esta crisis de expectativas tendría su origen en la estructura educacional donde se ha formado una nueva clase media, pero que ha tenido sus posibilidades laborales limitadas, en un contexto de alta desigualdad (Canales et al., 2020).

Probablemente las manifestaciones sociales en la vía pública han sido contenidas por la situación de emergencia sanitaria, sin embargo, sus elementos estructurales subyacen. Resulta relevante entonces, comenzar a comprender la experiencia de la pandemia, una emergencia sanitaria mundial, pero que se desenvuelve en condiciones sociopolíticas particulares en Chile: en medio de una revuelta popular, en un momento constituyente.

\section{Metodología}

Los resultados que se presentan a continuación son producto de dos procesos de levantamiento. Por una parte, se utilizan datos provenientes de un cuestionario web y, por otro lado, de la realización de un taller participativo cualitativo por video llamada. En este sentido, el análisis adopta un enfoque mixto que a partir del cuestionario en línea se propone medir magnitudes, pero al mismo tiempo intenta comprender las percepciones y experiencias subjetivas de las personas, para lo cual se utilizó el taller por video llamada. Este acercamiento mixto permite complementar las fortalezas de ambas metodologías y, de esta manera, tener una mayor comprensión de los datos recabados en un contexto muy limitado por la no presencialidad. Por lo tanto, es relevante reconocer los límites empíricos del presente artículo, destacando 
su aporte exploratorio durante los primeros meses de convergencia entre pandemia y estallido social.

El cuestionario fue desarrollado por el Instituto de Políticas Públicas de la Universidad Católica del Norte (UCN), sede Coquimbo, en colaboración con la Escuela de Ciencias Empresariales de la misma casa de estudios. Como se mencionó anteriormente, este instrumento fue de carácter no probabilístico y fue aplicado entre abril y mayo de 2020 a través de un formulario web auto administrado difundido a través de redes sociales. Los participantes pudieron acceder voluntariamente a contestar. Se aplicaron dos filtros, considerando como válidas solo las respuestas de personas que declararan vivir en alguna de las comunas de la Región de Coquimbo y fueran mayores de edad. Se respondieron de manera válida un total de 808 instrumentos. No se estimó un número de respuestas esperadas, como tampoco se definió una muestra, estrategia muestral o tasa de rechazo, debido al carácter dirigido del cuestionario, es decir, no probabilístico. Los resultados obtenidos fueron analizados con la aplicación de un ponderador por género de acuerdo con el Censo 2017 con el objetivo de tener una distribución más ajustada al universo regional. En el cuestionario se consideraron preguntas cerradas con categorías de respuesta nominales y ordinales sobre la evaluación de las medidas implementadas, los impactos, la confianza y las expectativas de futuro. Las preguntas fueron elaboradas por el equipo del Instituto, tomando como referencia la Encuesta IPP UCN 2018 y otras encuestas de opinión pública realizadas en Chile ${ }^{4}$. Los resultados fueron procesados a través de un análisis descriptivo considerando las limitaciones de un proceso no probabilístico el cual no permite realizar inferencias estadísticas.

Por otro lado, el presente artículo utiliza los resultados de un taller participativo cualitativo realizado también por el Instituto de Políticas Públicas de la UCN Coquimbo y que tuvo por objetivo construir un espacio de diálogo para conocer las distintas vivencias de las personas en el marco de la emergencia. Es importante mencionar que el taller tuvo como objetivo el levantamiento de información, y al mismo tiempo, el constituirse como un espacio de escucha (Canales, 2006) donde las personas pudieran expresar sus experiencias subjetivas y compartir con otras, en un contexto de aislamiento social. Para esta actividad se convocó de manera abierta a la comunidad (a través de medios digitales). El taller se desarrolló en el mes de agosto de 2020 por video llamada. Los participantes fueron divididos en 5 grupos (salas virtuales) contando con un facilitador/a. La técnica utilizada en el taller participativo fue de grupo focal, generando un diálogo a partir de preguntas realizadas por el facilitador/a de cada grupo. Las preguntas fueron administradas de manera semi-estructurada por lo cual hubo flexibilidad de adaptar éstas durante la ejecución de la entrevista, con el objetivo de construir con los y las participantes una perspectiva grupal (Hernández et al., 2014). La entrevista fue dirigida a partir

4 Como la Encuesta del Centro de Estudios Públicos (CEP) y la Encuesta Bicentenario de la Pontíficia Universidad Católica de Chile 
de tres preguntas centrales: ¿Cómo han vivido los tiempos de COVID-19 en los últimos meses? ¿Cuáles han sido sus emociones y pensamientos? ¿Cómo visualizan el futuro? y ¿Cuáles serán los impactos a mediano y largo plazo? Las sesiones fueron grabadas con la autorización de los y las asistentes para su posterior análisis. El procesamiento de la información se realizó a través de análisis de contenido el cual consiste, en palabras de Ruiz, "una técnica para leer e interpretar el contenido de toda clase de documentos" (2012: 92), que tiene un sentido simbólico o sentidos múltiples no siempre manifiestos. Como se mencionó anteriormente, el análisis de esta información recabada a través de la encuesta y el taller participativo no tiene un objetivo de representación, sino más bien exploratorio y de comprensión.

\section{Principales resultados}

\subsection{Principales resultados del cuestionario en línea}

De los diferentes resultados de la encuesta es posible destacar la evaluación que realizaron los encuestados respecto a las medidas implementadas por el gobierno en el período de la consulta tal como se observa en el gráfico $\mathrm{N}^{\mathrm{o}} 1$. Al respecto, la medida mejor evaluada es la instalación de aduanas y cordones sanitarios con $74 \%$ de las personas consultadas señalando esto como buena o muy buena. Un 52\% evalúa de manera positiva la creación de la mesa social. En el caso del toque de queda el 50\% está de acuerdo con esta medida, mientras que el 29\% la encuentra mala o muy mala. Un 58\% de los encuestados señala que las políticas económicas del Gobierno, tomadas hasta ese momento, son malas o muy malas. 


\section{Gráfico 1}

Evaluación de las medidas implementadas para enfrentar la pandemia

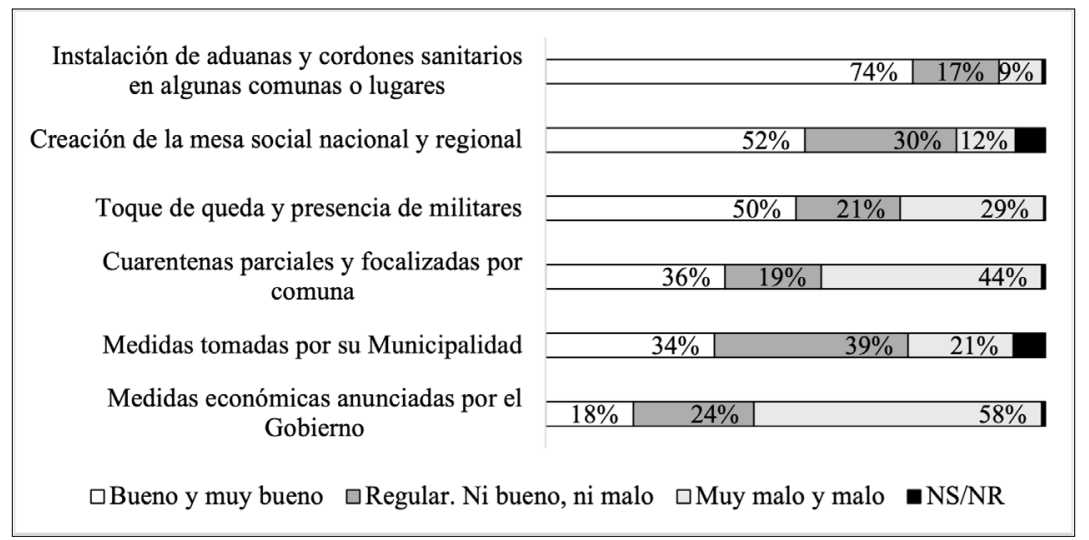

Fuente: Elaboración propia con base a resultados de encuesta IPP ECIEM UCN.

Sobre la confianza a las instituciones esta se encuentra muy debilitada, coincidiendo con otros estudios nacionales y regionales anteriores. Como se observa en el gráfico $\mathrm{N}^{\circ} 2$ las instituciones relacionadas al sistema político-democrático son las que obtienen una peor evaluación. Aquellas con mayores niveles de confianza o mucha confianza son los Centros de investigación (68\%), el Colegio Médico (64\%), las Universidades (49\%) y las radios (45\%). En cambio, los partidos políticos (1\%), el congreso $(2 \%)$, las empresas privadas $(9 \%)$ y la Iglesia Católica (10\%) son las que tienen los peores niveles. Destaca que un $71 \%$ declara desconfiar o tener mucha desconfianza del Gobierno de Chile. 


\section{Gráfico 2}

Nivel de confianza en las instituciones

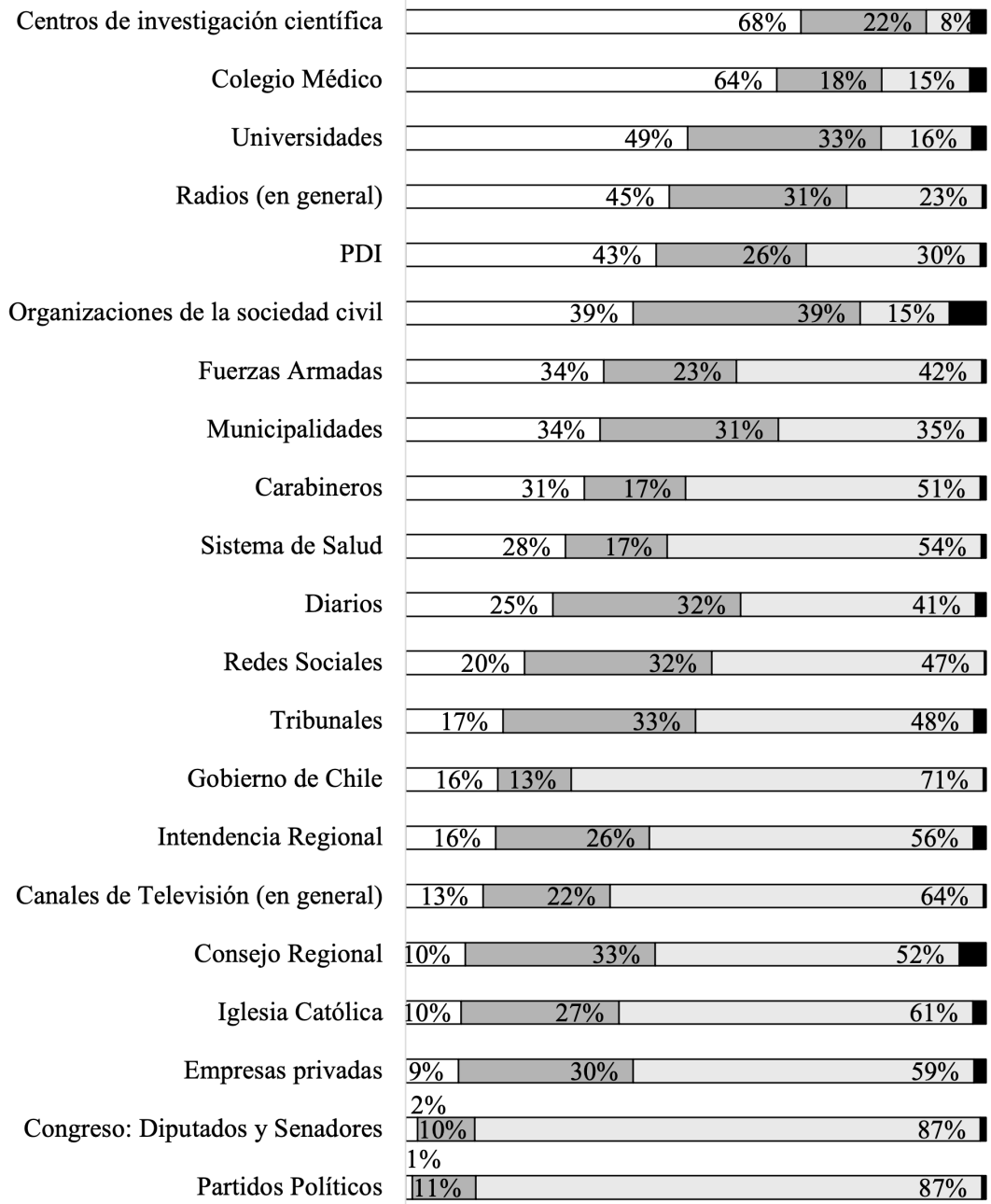

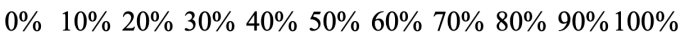

$\square$ Mucha confianza y confianza

$\square$ Mucha desconfianza y desconfianza $\square$ Indiferente, ni confianza ni desconfianza

-NS/NR

Fuente: Elaboración propia con base a resultados de encuesta IPP ECIEM UCN. 
Sobre la evaluación económica, un 83\% de los encuestados indica que la situación económica del país es mala o muy mala, sin embargo, se puede observar una opinión un poco más optimista respecto a la Región de Coquimbo, ya que el porcentaje de encuestados señala que la situación económica regional es mala o muy mala, se reduce al 57\%. Sin embargo, al momento de consultar por los efectos de la pandemia, un $97 \%$ considera que la pandemia afecta gravemente el empleo regional y un $95 \%$ lo cree para la economía.

El gráfico 3 muestra los resultados sobre la percepción de la situación económica futura (en los próximos 6 meses), el 74\% cree que la economía de la comuna será mala o muy mala, lo que aumenta levemente para la Región de Coquimbo y un poco más para el país. Un $77 \%$ de los encuestados señala que la situación económica futura de la región será mala o muy mala y un 85\% afirma esto para el país, sin embargo, este porcentaje se reduce al 33\% en el caso de la situación económica de la familia. Por otro lado, un $56 \%$ de los encuestados cree que los impactos económicos producto de la pandemia en la Región de Coquimbo continuarán más allá del 2020.

Gráfico 3

Percepción de la situación económica futura (6 meses)

\begin{tabular}{|c|c|c|c|}
\hline \multirow{3}{*}{$\begin{array}{l}\text { Futura situación económica de su familia } \\
\text { Futura situación económica de la Región de } \\
\text { Coquimbo }\end{array}$} & $19 \%$ & $44 \%$ & $33 \%$ \\
\hline & \multicolumn{3}{|l|}{$2 \%$} \\
\hline & \multicolumn{2}{|l|}{$16 \%$} & $77 \%$ \\
\hline \multicolumn{4}{|c|}{$2 \%$} \\
\hline Futura situación económica del país & $10 \%$ & & $85 \%$ \\
\hline \multicolumn{2}{|c|}{$\square$ Buena y muy buena $\quad \square$ Regular, ni mala ni buena } & $\square$ Mala y muy mala & $\square \mathrm{NS} / \mathrm{NR}$ \\
\hline
\end{tabular}

Fuente: Elaboración propia con base a resultados de encuesta IPP ECIEM UCN.

Respecto a los efectos personales, un $84 \%$ considera que la pandemia afecta gravemente su calidad de vida y un $70 \%$ cree que afecta su salud mental y/o emocional. Sin embargo, se observan diferencias por nivel socioeconómico, ya que dentro del grupo de personas con ingresos de hasta $\$ 464.000$, un $78 \%$ indica que la pandemia le ha afectado mental y emocionalmente, mientras que en el grupo de ingresos más altos, de $\$ 1.390 .000$ y más, esta respuesta llega a $63 \%$. 
En coherencia con lo anterior, un 78\% de los encuestados considera que tiene los recursos para implementar medidas de autocuidado, sin embargo, este porcentaje se reduce a un $49 \%$ en el grupo de personas con ingresos más bajos hasta \$464.000). Por otro lado, un $49 \%$ de los encuestados señala que sus ingresos han disminuido producto de la pandemia, sin embargo, lo más relevante de este aspecto es que este porcentaje es mayor entre las personas de niveles socioeconómicos más bajos. En este grupo la proporción sube, ya que un 76\% de quienes ganan hasta 464.000 señala que sus ingresos disminuyeron mientras que solo un $29 \%$ de quienes se encuentran sobre $\$ 1.390 .000$, indican una reducción. Respecto al hogar, un 25\% de las personas cree que la pandemia no les afectará en su situación económica, mientras un 25\% señala que, de contagiarse, tiene todos los recursos económicos para enfrentar la enfermedad y sus consecuencias. Sólo un 13\% cree que, si se contagia, el Estado le entregará el apoyo que necesite para enfrentar la enfermedad.

\section{Gráfico 4}

Impacto de la pandemia y/o posible enfermedad, a nivel personal y familiar

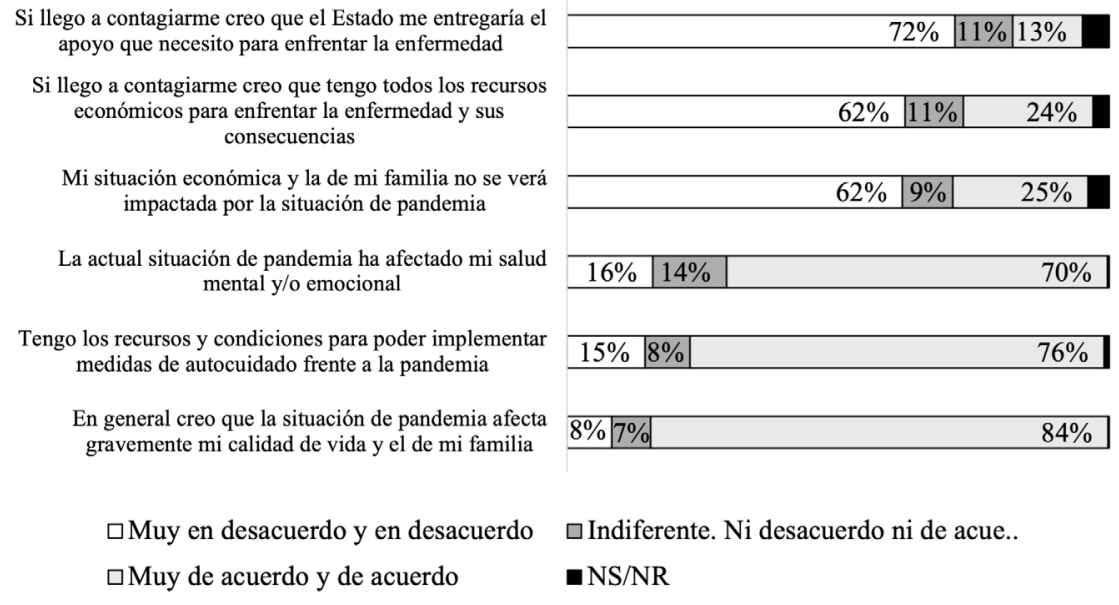

Fuente: Elaboración propia con base a resultados de encuesta IPP ECIEM UCN.

En la encuesta también fueron consultados los cambios que generará la pandemia. El gráfico 5 muestra los resultados sobre esta pregunta. Un 91\% cree que la pandemia conllevará grandes cambios en nuestros estilos de vida, un $85 \%$ estima que lo hará en las formas de relacionarnos socialmente. Un $63 \%$ en el sistema económico chileno y un $58 \%$ en el sistema de salud.Además, un $44 \%$ cree que el llamado "distanciamiento 
social" se deberá sostener más allá del 2020, mientras un $54 \%$ pensaba que esta medida sería necesaria un tiempo inferior.

\section{Gráfico 5}

Impactos sociales futuros. Nivel de acuerdo con la frase "La situación de pandemia generará grandes cambios en...”

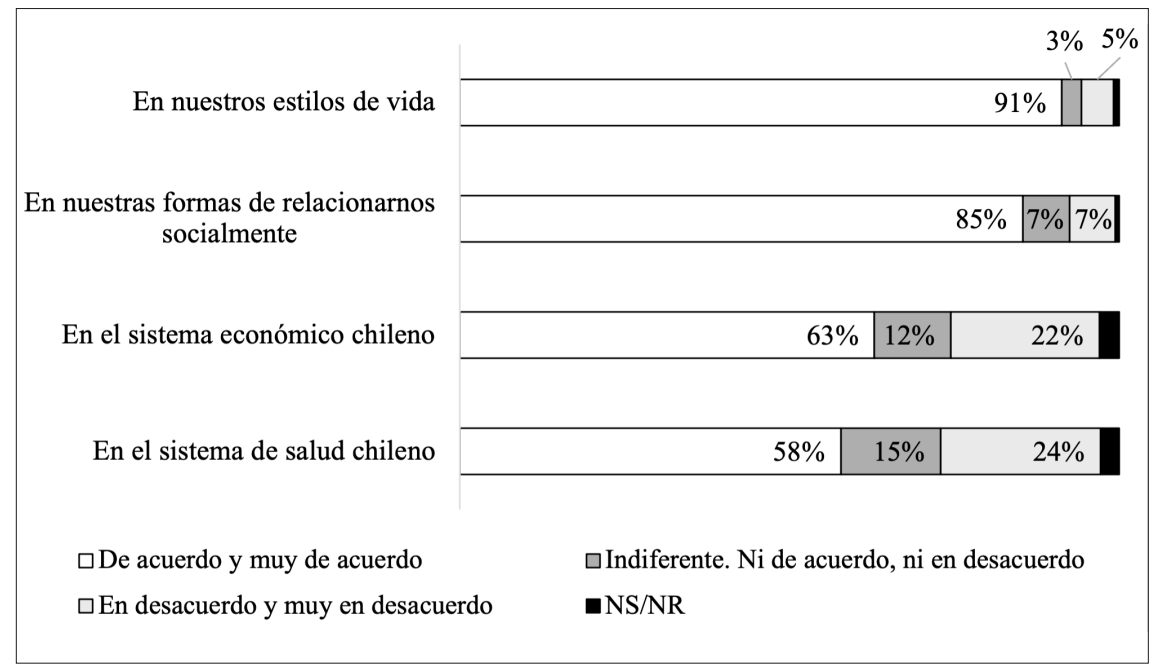

Fuente: Elaboración propia con base a resultados de encuesta IPP ECIEM UCN.

Finalmente, un 65\% de los encuestados cree que las movilizaciones sociales en la Región de Coquimbo se agudizarán y se incrementarán respecto al 2019, una vez finalizadas las medidas de distanciamiento social y la emergencia sanitaria.

\subsection{Principales resultados del taller participativo}

\subsubsection{Seguir funcionando y re-habilitar el espacio privado (quienes pueden)}

En general los y las participantes del diálogo señalan estar desde marzo en casa guardado "cuarentena". Quienes han podido teletrabajar se muestran agradecidos por esta posibilidad, manteniendo salidas del hogar para la realización de compras o trámites impostergables. Destaca esta valoración positiva del "teletrabajo" en cuanto que se reconoce como una modalidad que no es factible para todos y por lo tanto les permite seguir "funcionando" o "cumpliendo" con las actividades laborales. La experiencia es diferente para quienes deben continuar sus tareas de manera presencial, quienes hacen énfasis en el miedo al contagio y en seguir "los protocolos" para evitarlo. Algo similar ocurre con aquellos que, estando en el hogar, 
tienen algún miembro del grupo cumpliendo funciones presenciales: en la medida que existe un canal de contacto con el exterior, es plausible de contagio.

De acuerdo con lo anterior, se puede observar el establecimiento de una nueva frontera entre el hogar, y el exterior, donde el hogar aparece como el espacio seguro y a la vez funcional, mientras el exterior representa amenazas. Por otro lado, ese espacio exterior no es solamente la comuna o el país, sino que "el mundo", describiéndose la pandemia como algo transversal y que, por lo tanto, acerca la realidad internacional al espacio cotidiano.

La cotidianidad que se vivía en el exterior, antes de la pandemia, se traslada al interior del hogar lo cual, según los y las participantes, ha tenido un impacto "mental” ya que se deben realizar múltiples tareas en el mismo espacio que, anteriormente, tenía otro objetivo. Trabajar, cuidar a los hijos/as y sus actividades escolares y adicionalmente realizar las labores domésticas es descrito como algo dificil de compatibilizar, especialmente para las mujeres que trabajan remuneradamente con teletrabajo y, además, realizan las tareas del cuidado, sin poder limitar o salir de esta dinámica permanente de funcionalidad. Como señala una de las participantes, esto "le da la sensación de volverse loca, con la angustia de no poder salir". Sin embargo, algunos participantes plantean este periodo como un aprendizaje para ser más solidario en compartir las labores del hogar, para que, por una estructura de rol anteriormente determinada, no siguiera recayendo en una sola persona. El teletrabajo ha significado, además, re-educarse en tecnologías para poder trabajar, pero también para poder comunicarse con otros. La distancia física con familiares es uno de los aspectos más lamentados, al mismo tiempo que se reconoce un proceso de aprendizaje con las personas en el hogar incluso indicando que ha sido una oportunidad de "encuentro" con los otros miembros del núcleo.

\subsubsection{La incertidumbre permanente}

Se señala este período como un "tiempo duro", especialmente respecto a la salud mental planteándose que el encierro comienza a ser insostenible. Se indica que existe angustia y frustración, especialmente por no poder salir. Sin embargo, comentan que se ha transitado por muchas emociones en este tiempo, siendo una "montaña rusa de emociones". Algunas personas han sentido pena y angustia, miedo a la enfermedad por la propia salud, pero también miedo por los familiares, específicamente por padres y abuelos (personas mayores como principal grupo "vulnerable"). Por otro lado, se hace mención la sensación de impotencia por no poder ayudar o asistir a un familiar, especialmente si se encuentra contagiado por la enfermedad.

Durante los diálogos se hace constante referencia a la necesidad de adaptación y la incertidumbre como parte del nuevo escenario. Se debe "aprender a convivir" con la pandemia, lo cual, según los y las participantes, es algo nuevo ya que anteriormente "uno estaba acostumbrado a manejar en algún nivel los escenarios", sin embargo, actualmente hay incertidumbre de lo que va a pasar mañana, de los impactos o 
nuevas consecuencias que no se han manifestado. Se habla de una sensación de "irrealidad": pese a que, han pasado varios meses desde el inicio de la crisis, se describe la percepción de "vivir en una película de ciencia ficción”. En este sentido, se habla de un contexto excepcional, como un detonador de "lo mejor o peor de las personas".

La pandemia acerca entonces la contingencia: todo puede cambiar en cualquier momento y, por lo tanto, la preocupación debe ser por el presente. Los y las participantes se perciben como vulnerables al riesgo de contagiarse, perder la vida, perder a otros o perder las condiciones de vida que actualmente tienen.

\subsubsection{El contagio y los otros}

El contagio es percibido con miedo, como una posibilidad atemorizante en el exterior pero que, además, "ingresa" al hogar, en la medida que hay personas que "salen" o que las mismas personas deben salir. Otro elemento que destaca es la referencia al virus como algo "que está en todos lados", pero que al mismo tiempo "no se sabe dónde está". A diferencia de otros riesgos o amenazas el virus es invisible y por lo tanto se percibe como algo omnipresente frente a lo cual no se puede hacer lo suficiente.

Respecto a la experiencia de haberse contagiado o de haber tenido que guardar cuarentena por contacto estrecho, se describe esta experiencia como una vivencia solitaria y de decepción por quienes se alejaron (amistades) como también se manifiesta haber sentido temor por revelar esta situación por miedo al rechazo. Se cuestiona que "otros" no se cuidan, hacen eventos, no utilizan bien la mascarilla y no aplican las medidas necesarias. Sin embargo, al mismo tiempo, se reconoce una situación desigual para enfrentar la crisis y, en este sentido, algunos dicen sentirse "privilegiados" y aliviados de que esta crisis sanitaria les haya tocado en esta etapa de la vida, con una situación económica estable y con la posibilidad de cuidar a los hijos o hijas. En este sentido se menciona que existiría una mayor conciencia social por la situación de los otros y una mayor necesidad de colaborar con acciones en conjunto, en la medida que el fin de la pandemia requiere de las acciones de todos. Pese a lo anterior, se señala que esta colaboración colectiva es improbable, ya que se describe la sociedad actual como individualista

En relación con lo anterior, algunas personas que pasaron por la situación de contagio destacan la solidaridad de conocidos y amigos que les brindaron apoyo a través de la entrega de ayudas económicas o alimentos. Sin embargo, se menciona la sensación de vergüenza y culpa por recibir ayuda sabiendo que otros podrían necesitarla más. Se describe una sensación de ofensa a la "dignidad" pero que se resuelve reconociendo la necesidad: "la dignidad no le va a quitar el hambre". 


\subsubsection{Los cambios y el futuro}

La pandemia se percibe como un momento o período que tendría distintos impactos, los y las participantes reiteran la existencia de un cambio de "paradigma" hacia uno donde se debe aprender a vivir el ahora, dejando las estructuras y planificaciones. En este sentido se reconoce que antes de la pandemia predominaba planificar y proyectar el futuro, dejando de lado el goce del presente, especialmente respecto al espacio privado y las relaciones familiares. La pandemia de esta forma vendría a colocar la cotidianidad al centro, revalorizándose las relaciones familiares y el goce más inmediato. Según los y las participantes, este período ha sido de aprendizaje tanto en lo personal como en lo social donde se destaca el desarrollo de un sentido más solidario y consciente de las desigualdades.

El futuro, incierto, se observa con desconfianza, no se visualiza la posibilidad de "volver a lo que era antes" ya que se cree que el virus seguirá estando "ahí" y se comenta que es mejor optar por no tener expectativas temporales respecto al futuro y el término de esta crisis, y más bien vivir el día a día.

Ya que el virus continuará existiendo, como amenaza invisible, deberán continuar las medidas, especialmente aquellas relacionadas a la interacción social. Este aspecto es señalado como uno de los más complejos, en la medida que, según los participantes, será dificil volver a tener contacto con las otras personas. Se señala que probablemente se deberá ser más selectivos al momento de reunirse con otros, reduciendo los grupos a familiares y amigos más cercanos. Se visualiza que el teletrabajo se instalará como nueva modalidad, y también se aplicará en el ámbito educacional. Quienes se dedican a otras tareas, como la cultura y las artes, por ejemplo, deberán "reinventarse" para el uso permanente de plataformas digitales.

Se indica que el país ha estado enfrentando muchos cambios y, por lo tanto, no se volverá a la "normalidad". De esta forma se señalan que la vida para muchas personas es difícil y que con la pandemia el futuro podría ser más difícil aún. Existe una preocupación por la pobreza, el desempleo y las migraciones, y manifestando la necesidad de una mayor distribución de la riqueza, de "potenciar" los emprendimientos para aquellas personas que perdieron su trabajo y promover una mayor incorporación de la mujer y los niños/as en los procesos. En este marco, son partidarios de que se debe hacer un trabajo intersectorial y con descentralización para políticas públicas adecuadas. También mencionan la falta de liderazgos que requiere esta crisis, "claros, potentes y con sabiduría" sobre todo por la necesidad de adaptarse a nuevos otros escenarios como el cambio climático. En este sentido los participantes ven esta actual crisis como una oportunidad, en la medida que la ilusión de controlar los escenarios se ha desvanecido y por lo tanto "se debe aprender a lidiar con la incertidumbre y ser optimista". 


\section{Síntesis de resultados}

De acuerdo con los indicadores, es posible observar que las medidas implementadas ${ }^{5}$, son evaluadas de manera regular, siendo aquellas económicas las que reciben peor evaluación. Esto es congruente con que, para las personas participantes, el impacto económico es muy relevante, destacándose que la percepción sobre este ámbito es peor para el país y la región que para la propia experiencia familiar. La percepción de que la situación económica del hogar es mejor que el país o la región, es coherente con la descripción del hogar como el espacio más seguro en el actual contexto, frente al exterior, que es descrito como espacio de amenaza de acuerdo con los resultados cualitativos. Al mismo tiempo, los resultados cualitativos permiten observar que las personas se reconocen en una situación favorecida frente a otras realidades, lo cual es coherente con evaluar la situación económica de otros como peor que la propia.

Por otro lado, esta percepción negativa de la economía actual, también se proyecta hacia el futuro, lo que se podría relacionar con la percepción de inestabilidad e incertidumbre que en los talleres se indica "llegó para quedarse". Los participantes en general sienten que la pandemia les afectará económicamente y perciben no tener suficientes recursos para enfrentar una situación de contagio. Destaca que la gran mayoría de las personas considera que el Estado no le brindaría los apoyos necesarios para enfrentar la enfermedad. Lo anterior, podría relacionarse con la sensación de vulnerabilidad descrita en los talleres donde se narra como un imperativo el "seguir funcionando" en la medida que son los propios recursos y herramientas los únicos disponibles para enfrentar los adversos escenarios.

Se reconocen importantes impactos de la pandemia sobre todo en la calidad de vida y en la salud mental y emocional. La incorporación de las actividades públicas al espacio privado, la soledad, la incertidumbre y la percepción de un escenario "irreal" ha significado un reordenamiento no sólo de dinámicas cotidianas, sino que también de las relaciones con los otros y de los proyectos de futuro. Esto se podría relacionar con que, según los encuestados, la pandemia tendrá principalmente efectos en las formas de relacionarnos y estilos de vida. El futuro, incierto, aparece igualmente con demandas de mayor equidad y la necesidad de implementar medidas para quienes las necesitan, lo cual a su vez podría explicar la proyección de nuevas movilizaciones sociales en el futuro.

En el taller se hace referencia a la necesidad de nuevos liderazgos, lo cual podría relacionarse con la importante desconfianza expresada a las instituciones, de manera general, pero especialmente respecto a las instituciones políticas democráticas donde la confianza es prácticamente inexistente. Por otro lado, las instituciones con mejores

Evaluación realizada a las medidas en el momento del levantamiento de información, primer semestre 2020. 
niveles de confianza son las relacionadas a la ciencia y el conocimiento, lo cual es coherente con que las medidas mejor evaluadas relacionadas con estos actores.

\section{Discusión}

A continuación, se intentará discutir los resultados de los levantamientos a la luz de la literatura revisada. Es importante señalar los límites de este ejercicio de articulación entre estallido social y pandemia, tanto por limitaciones metodológicas, como también por la complejidad de ambos procesos que sobrepasan las posibilidades de este artículo. En este sentido, es relevante insistir en el aporte exploratorio de esta reflexión.

Como se señaló, el estallido social tiene causas complejas, entre las cuales se pueden señalar demandas por libertades, derechos sociales, económicos, justicia e igualdad, entre otras necesidades y garantías insatisfechas (Brieba, 2020; Jiménez-Yáñez, 2020), construcciones interrelacionales del sujeto consigo mismos, los otros y la sociedad (Araujo \& Benítez, 2019; Canales en Hopenhayn, 2020; Morales, 2020) y crisis estructurales relacionadas al sistema político democrático (Heiss, 2017; Miranda, 2018; Morales, 2020). Los anteriores elementos se relacionan con la confianza, ya sea racional, cultural (Saldaña \& Pineda, 2019) o como expresión de apoyo político (Easton, 1975) la cual tiene un rol relevante frente a los riesgos para reducir las complejidades de la incertidumbre (Beck, 1998).

De acuerdo con los levantamientos de información realizados, los cuales coinciden con otros recientes instrumentos de opinión pública (CEP, 2021), es posible señalar que la desconfianza permanece en un escenario de riesgo como la pandemia y la incertidumbre, mientras los cambios, se convierten (paradójicamente) en la principal certeza.

Respecto a las demandas o necesidades insatisfechas, los resultados muestran evaluaciones negativas sobre el presente y el futuro económico, lo cual es coherente con la percepción de incertidumbres y podrían explicar la desconfianza como producto de una evaluación racional frente a demandas, garantías y necesidades insatisfechas, siguiendo la teoría institucional racional (Saldaña \& Pineda, 2019). Sin embargo, las evaluaciones materiales personales tienden a ser mejores que las respecto a otros. De hecho, en las entrevistas, se reconoce como "privilegio" contar con empleo o poder teletrabajar y estar mejor, en comparación a otros. Existe un fuerte relato sobre los "otros" que se encuentran con necesidades críticas. En este sentido podríamos decir que la insatisfacción no es (al menos exclusivamente) personal, sino más bien social. En otras palabras, siguiendo la teoría de la confianza como evaluación racional, una valoración satisfactoria de la propia realidad parece ser insuficiente para la confianza, si se evidencian insatisfacciones de otros. 
Respecto a las relaciones con los otros, las personas perciben un importante impacto de la pandemia en lo relacional. Si la confianza deviene de la interacción con otros, especialmente de otros diferentes (Putman, 1994), se podría suponer que el aislamiento no favorecería a la recomposición de estos vínculos, permaneciendo o incluso agudizando la desconfianza interpersonal. Por el contrario, los otros y el exterior se describen como peligrosos y el espacio que se describe como seguro y confiable, es el hogar con el círculo cercano familiar. Los riesgos reconfiguran las fronteras con el mundo, señala Beck (1998), pero también parece ser que redefinen los límites del espacio privado. Esta percepción es coherente además con una mirada centrada en el mérito propio para enfrentar los riesgos. El énfasis en "seguir funcionando" ilustra la importancia del esfuerzo personal, lo que Douglas y Wildavsky (1983) han definido como sociedad de mercado, con una perspectiva del riesgo de corto plazo y donde se depende de las acciones de los individuos. Sin embargo, aquí es posible observar una interesante articulación pues pese a que los otros son riesgo y el foco de la seguridad está en el espacio privado, como se mencionó anteriormente, hay una preocupación por los otros pese a que no haya interacción con ellos.

Sobre las instituciones, destaca la baja y casi nula confianza de manera general, pero, sobre todo, respecto a las instituciones políticas democráticas. Esto se podría relacionar con la evaluación negativa que se hace de las medidas. Sin embargo, como señala Easton (1975), la confianza como parte del apoyo político se construye en el tiempo y por lo tanto, la desconfianza y la evaluación negativa podría devenir por lo que las instituciones representan en el marco del estallido social, más que por lo que hacen. No es menor destacar que las instituciones científicas o relacionadas a la ciencia tienen los mejores niveles confianza, lo que coincide con estudios anteriores (CEP, 2019; Instituto de Políticas Públicas, 2019; ACTIVA, 2020).

De esta forma, se puede observar que la pandemia en Chile se experimenta desde la percepción de desprotección y vulnerabilidad. Lo anterior, a modo de hipótesis, podría ser más que una evaluación racional, una experiencia devenida de procesos anteriores. Para las personas participantes, la pandemia profundiza las desigualdades sociales que han sido cuestionadas por la movilización social y la desconfianza en las instituciones no se logra reestablecer, sino que, más bien por el contrario, se mantiene sostenidamente o incluso se profundiza. Si el "estallido social" ha significado un proceso de transformación sociopolítico, es importante destacar que éste se desarrolla en un contexto en el cual las personas se perciben vulnerables y desprotegidas, especialmente respecto al Estado. Sin confianzas, queda el riesgo y éste aparece como parte de la nueva realidad, algo a lo cual "hay que adaptarse". Queda por supuesto abierta la pregunta de qué implicancias tendrá esta experiencia en los procesos sociales y políticos venideros. 


\section{Referencias}

ACTIVA (2020, abril). Pulso Ciudadano: Primera Quincena de abril 2020. Somos Activa. https:// chile.activasite.com/page/3/?s\&tipo_de_estudio=pulso-ciudadano

Albala, A. \& Vieira, S. M. (2014). ¿Crisis de los partidos en América Latina? El papel de los partidos políticos latinoamericanos en el escenario reciente. Política. Revista de Ciencia Política, 52(1), 145-170. https://doi.org/10.5354/rp.v52i1.33102

Alemán, J. (2013). De la sociedad del riesgo al desmantelamiento del estado de bienestar. Dilemata, 11, 139-147.

Araujo, K. \& Benítez, F. (2019).De incertidumbres, investigación y anclajes socio-existenciales: Una entrevista con Kathya Araujo tras el acontecer de octubre. Cuadernos de Teoría Social, 5(10), 151-170.

Beck, U. (1998). La sociedad del riesgo: Hacia una nueva modernidad. Paidós.

Biblioteca del Congreso Nacional (2019, noviembre 15). Acuerdo Por la Paz Social y la Nueva Constitución. bcn.cl; BCN. Biblioteca del Congreso Nacional de Chile. https://www.

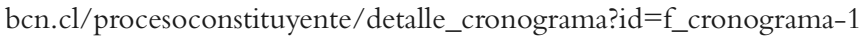

Brieba, D. (2020). El estallido social en Chile desde el igualitarismo relacional de Elizabeth Anderson. Revista de Sociología, 35(1), 31. https://doi.org/10.5354/0719529X.2020.58105

Canales, M. (Ed.). (2006). Metodologías de investigación social (1ra Edición). LOM.

Canales, M., Guajardo, F. \& Orellana, V. (2020). La elite del llano: De la promesa a las desilusiones en la trayectoria postsecundaria de los jóvenes de la nueva clase media. Última década, 28(53), 78-102. https://doi.org/10.4067/S0718-22362020000100078

CEP (2019). Estudio Nacional de Opinión Pública No84, diciembre 2019-Centro de Estudios Públicos. https://www.cepchile.cl. https://www.cepchile.cl/cep/encuestas-cep/ encuestas-2010-2019/estudio-nacional-de-opinion-publica-n-84-diciembre-2019

CEP (2021). Encuesta especial CEP, abril 2021-Centro de Estudios Públicos. https://www. cepchile.cl. https://www.cepchile.cl/cep/encuestas-cep/encuestas-2010-2019/1encuesta-especial-cep-abril-2021

Choudhry, S. (2008). Ackerman's higher lawmaking in comparative constitutional perspective: Constitutional moments as constitutional failures? Icon-International Journal of Constitutional Law, 6(2), 193-230. https://doi.org/10.1093/icon/mon002

Comisión Económica para América Latina y el Caribe (2021). Panorama Social de América Latina 2020. CEPAL. https://www.cepal.org/es/publicaciones/46687-panoramasocial-america-latina-2020 
Douglas, M. \& Wildavsky, A. (1982). Risk and Culture: An Essay on the Selection of Technological and Environmental Dangers (1. ${ }^{a}$ ed.). University of California Press. https://www.jstor. org/stable/10.1525/j.ctt7zw3mr

Easton, D. (1975). A Re-assessment of the Concept of Political Support. British Journal of Political Science, 5(4), 435-457. https://doi.org/10.1017/S0007123400008309

Heiss, C. (2017). Legitimacy crisis and the constitutional problem in Chile: A legacy of authoritarianism. Constellations - An International Journal of Critical and Democratic Theory, 470-479. https://doi.org/10.1111/1467-8675.12309

Hernández, R., Fernández, C. \& Baptista, M. del P. (2014). Metodología de la investigación (6ta edición). Mc Graw Hill.

Hopenhayn, D. (2020, agosto 29). Manuel Canales, sociólogo: "Para encauzar el estallido hay que interpretar su esperanza, no sólo su rabia”. La Tercera. https://www.latercera.com/la-terceradomingo/noticia/manuel-canales-sociologo-para-encauzar-el-estallido-hay-queinterpretar-su-esperanza-no-solo-su-rabia/QS25PVQAVBAEPNIENG2RSY7O2M/

Instituto de Políticas Públicas (2019). Encuesta IPP Coquimbo | Instituto Políticas Públicas del Norte. https://www.politicaspublicasdelnorte.cl/encuesta-ipp-coquimbo/

Instituto Nacional de Derechos Humanos (2020). Reporte de datos 18 de febrero de 2020.

Jiménez-Yáñez, C. (2020). \#Chiledespertó: Causas del estallido social en Chile. Revista Mexicana de Sociologia, 82(4).

Lewis, J. D. \& Weigert,A. (1985).Trust as a Social Reality. Social Forces, 63(4), 967-985. https:// doi.org/10.2307/2578601

Miranda, R. (2018). Democracia de alta calidad en América Latina. Análisis histórico comparado entre Chile, Costa Rica y Uruguay. Revista de Ciencia Política, 56, 89-106. https://doi.org/doi:10.5354/0716-1077.2018.58135

Mitjavila, M. (1999). El riesgo y las dimensiones institucionales de la modernidad. https://www. colibri.udelar.edu.uy/jspui/handle/20.500.12008/6792

Morales, M. (2008). Evaluando la confianza institucional en Chile: Una mirada desde los resultados LAPOP. Revista de ciencia política (Santiago), 28(2), 161-186. https://doi. org/10.4067/S0718-090X2008000200007

Morales, M. (2020). Estallido social en Chile 2019: Participación, representación, confianza institucional y escándalos públicos. Análisis Político, 33(98), 3-25. https://doi. org/10.15446/anpol.v33n98.89407

Programa de las Naciones Unidas para el Desarrollo (2015). Mecanismos de cambio constitucional en el mundo. UNDP. https://www.cl.undp.org/content/chile/es/home/library/ democratic_governance/mecanismos-de-cambio-constitucional-en-el-mundo.html 
Putman, R. (1994). Para hacer que la democracia funcione: La experiencia italiana en descentralización administrativa. Editorial Galac.

Ruiz, J. I. (2012). Metodología de la investigación cualitativa. https://www-digitaliapublishingcom.ezproxy2.ucn.cl/a/15155/metodologia-de-la-investigacion-cualitativa

Saldaña,V. \& Pineda, M. (2019). Confianza en instituciones políticas: Factores que explican la percepción de confianza en Chile. Revista Temas Sociológicos, 25, 231-258. https://doi. org/10.29344/07196458.25.2169

Spickard, J. V. (1989). A Guide to Mary Douglas's Three Versions of Grid/Group Theory. Sociological Analysis, 50(2), 151-170. https://doi.org/10.2307/3710986

Tamayo, M. \& Carrillo, E. (2003). Políticas públicas y apoyo político en el Estado de las Autonomías. Gestión y Análisis de Políticas Públicas. Nueva época.https://doi.org/10.24965/ gapp.vi26-27.348

Taylor-Gooby, P. \& Zinn, J. O. (2006). Current directions in risk research: New developments in psychology and sociology. Risk Analysis: An Official Publication of the Society for Risk Analysis, 26(2), 397-411. https://doi.org/10.1111/j.1539-6924.2006.00746.x

United Nations (2021). 2020 Report of the Secretary-General on the Work of the Organization | United Nations. Annual Report. https://www.un.org/annualreport/

Urteaga, E. (2013). La teoría del capital social de Robert Putnam: Originalidad y carencias. Reflexión Politica, 15(29), 44-60.

World Health Organization (2021). Coronavirus Disease (COVID-19) Situation Reports. https:// www.who.int/emergencies/diseases/novel-coronavirus-2019/situation-reports

Enviado: 02 de mayo de 2021

Aceptado: 02 de julio de 2021 
